\title{
Urine 5MedC, a Marker of DNA Methylation, in the Progression of Chronic Kidney Disease
}

\author{
Akifumi Onishi, ${ }^{1,2}$ Hitoshi Sugiyama ${ }^{D},{ }^{2}$ Masashi Kitagawa, ${ }^{1}$ Toshio Yamanari, ${ }^{1}$ \\ Keiko Tanaka, ${ }^{1,3}$ Ayu Ogawa-Akiyama, ${ }^{1}$ Yuzuki Kano, ${ }^{1}$ Koki Mise, ${ }^{1}$ Katsuyuki Tanabe $\mathbb{D}^{1},{ }^{1}$ \\ Hiroshi Morinaga, ${ }^{4}$ Masaru Kinomura, ${ }^{1}$ Haruhito A. Uchida $\left(\mathbb{D},{ }^{5}\right.$ and Jun Wada ${ }^{1}{ }^{1}$ \\ ${ }^{1}$ Department of Nephrology, Rheumatology, Endocrinology and Metabolism, Okayama University Graduate School of Medicine, \\ Dentistry and Pharmaceutical Sciences, Okayama, Japan \\ ${ }^{2}$ Department of Human Resource Development of Dialysis Therapy for Kidney Disease, Okayama University Graduate School \\ of Medicine, Dentistry and Pharmaceutical Sciences, Okayama, Japan \\ ${ }^{3}$ Department of Molecular Life Sciences, Tokai University School of Medicine, Kanagawa, Japan \\ ${ }^{4}$ Division of Medical Informatics, Okayama University Hospital, Okayama University Graduate School of Medicine, Dentistry and \\ Pharmaceutical Sciences, Okayama, Japan \\ ${ }^{5}$ Department of Chronic Kidney Disease and Cardiovascular Disease, Okayama University Graduate School of Medicine, \\ Dentistry and Pharmaceutical Sciences, Okayama, Japan
}

Correspondence should be addressed to Hitoshi Sugiyama; hitoshis@okayama-u.ac.jp

Received 9 January 2019; Revised 2 April 2019; Accepted 2 June 2019; Published 1 July 2019

Guest Editor: Chia-Ter Chao

Copyright ( 2019 Akifumi Onishi et al. This is an open access article distributed under the Creative Commons Attribution License, which permits unrestricted use, distribution, and reproduction in any medium, provided the original work is properly cited.

\begin{abstract}
Background. Alterations in DNA methylation may be involved in disease progression in patients with chronic kidney disease (CKD). Recent studies have suggested that 5-methyl-2' -deoxycytidine (5MedC) may be a marker of hypermethylation of DNA. Currently, there is no information available regarding the urine levels of $5 \mathrm{MedC}$ and its association with the progression of CKD. Method. We examined the urine levels of $5 \mathrm{MedC}$ in spot urine samples from 308 patients with CKD (median age: 56 years, male: $53.2 \%$, and glomerulonephritis: $51.0 \%$ ) using a competitive enzyme-linked immunosorbent assay and investigated the relationships among urine $5 \mathrm{MedC}$, urine albumin, urine $\alpha 1$-microglobulin $(\alpha 1 \mathrm{MG})$, and the laboratory parameters associated with CKD. The patients were followed for three years to evaluate renal endpoints in a prospective manner. Results. The urine 5MedC level was significantly increased in the later stages of CKD compared to the early to middle stages of CKD. In multiple logistic regression models, urine $5 \mathrm{MedC}$ was significantly associated with the prediction of later CKD stages. Urine $5 \mathrm{MedC}$ (median value, $65.9 \mu \mathrm{mol} / \mathrm{gCr}$ ) was significantly able to predict a $30 \%$ decline in the estimated GFR or a development of end-stage renal disease when combined with macroalbuminuria or an increased level of urine $\alpha 1 \mathrm{MG}$ (median value, $5.7 \mathrm{mg} / \mathrm{gCr}$ ). Conclusion. The present data demonstrate that the urine $5 \mathrm{MedC}$ level is associated with a reduced renal function and can serve as a novel and potent biomarker for predicting the renal outcome in CKD patients. Further studies will be necessary to elucidate the role of urine DNA methylation in the progression of CKD.
\end{abstract}

\section{Introduction}

Epigenetic changes are stable and heritable but reversible modifications, including DNA methylation, posttranscriptional modifications of histone, and remodeling of chromatin $[1,2]$. Among them, DNA methylation is a crucial epigenetic alteration observed in eukaryotic organisms and has been shown to be associated with many biological and cellular processes, such as embryonic development, transcription, structure of chromatin, and stability of chromosome [3, 4]. Recently, several human diseases have been reported to be associated with abnormal DNA methylation $[4,5]$.

Chronic kidney disease (CKD) is a significant concern given the increasing number of such patients throughout 
the entire world [6]. CKD is characterized by either or both a glomerular filtration rate (GFR) less than $60 \mathrm{~mL} / \mathrm{min} / 1.73 \mathrm{~m}^{2}$ and signs of kidney injury of at least 3 months' duration $[6,7]$. A reduced estimated GFR (eGFR) and severe degree of albuminuria independently predict end-stage renal disease and mortality in CKD patients [8]. There is an urgent need to identify novel biomarkers in patients with $\mathrm{CKD}$ in order to better detect those at high risk of a rapid decline in the renal function so that effective therapies can be used to inhibit the disease progression [9].

A recent large-scale genome-wide evaluation of DNA methylation showed that DNA hypomethylation and hypermethylation were present at different loci in patients with CKD [10]. DNA hypermethylation in the peripheral blood may be linked to inflammation possibly associated with bacterial infections in $\mathrm{CKD}$ patients with incident dialysis [11].

5-Methyl-2' -deoxycytidine (5MedC) (PubChem CID: $440055)$ is a product of the base excision repair (BER) and nucleotide excision repair (NER) pathways of active DNA methylation. $5 \mathrm{MedC}$ was detected in the urine of healthy individuals as well as in those with certain diseases via several methods [12-16]; however, little is known about its levels in the urine of CKD patients or its association with progression of disease in such patients.

We therefore determined the urine levels of $5 \mathrm{MedC}$ and its association with progression and renal outcome in patients with CKD.

\section{Methods}

2.1. Study Design. The study subjects were outpatients who had visited the Division of Nephrology in Okayama University Hospital between February 2009 and February 2012. All patients were diagnosed with CKD in accordance with the eGFR and the presence of kidney damage, as characterized by the National Kidney Foundation K/DOQI Guideline [6]. The eGFR was calculated as described previously [17]. Hypertension was defined as described previously $[18,19]$. The mean blood pressure (MBP) was calculated as diastolic blood pressure + (systolic blood pressure - diastolic pressure)/3.

All procedures in the current study were performed according to national and institutional ethical guidelines of human studies and guidelines in the Declaration of Helsinki. The ethics committee of Okayama University Graduate School of Medicine, Dentistry and Pharmaceutical Sciences approved the study (KEN1607-010). All subjects gave written informed consent.

A prospective and longitudinal study was performed to investigate the relationship among urine 5MedC levels, clinical parameters, and the renal outcome in CKD patients. The patients who participated in this study were recruited between February 2009 and February 2012. Patients were followed for up to 3 years, but those who were followed for less than 3 months $(n=51)$ or who started renal replacement therapy within 3 months of the participation $(n=5)$ were excluded. As a result, a total of 308 patients were included in the analysis. Of these patients, 199 patients (male 107, female 92) were overlapped with the previous study [19]. In accordance with the established protocol, we excluded any patients with established atherosclerotic complications (congestive heart failure, coronary artery disease, or peripheral vascular disease) [19]. Patients with infection, acute kidney injury, cancer, and Alzheimer's disease at entry were also excluded.

2.2. Laboratory Measurement of Urine Biomarkers. Spot urine samples were collected from patients in the morning, as described previously [19]. The urine 5MedC levels were measured using a Global DNA Methylation Enzyme-Linked Immunosorbent Assay (ELISA) Kit (Cell Biolabs Inc., San Diego, CA, USA), which was a competitive enzyme immunoassay developed for the rapid quantitation and detection of $5 \mathrm{MedC}$ in urine directly. The quantity of $5 \mathrm{MedC}$ in an unknown sample is calculated by comparing its absorbance with that of a known $5 \mathrm{MedC}$ standard curve. The kit has a $5 \mathrm{MedC}$ detection sensitivity range of $150 \mathrm{nM}$ to $10 \mu \mathrm{M}$. The concentration of 8 -hydroxy-2' ${ }^{\prime}$-deoxyguanosine (8-OHdG) in urine samples was also determined using an ELISA kit (R\&D Systems, Minneapolis, MN, USA) as previously described [20, 21].

The median duration of storage between collection of urine and measurement of biomarkers was 48 months (interquartile range, 34-49 months). The urine levels of albumin, total protein, creatinine $(\mathrm{Cr})$, and alpha1-microglobulin $(\alpha 1 \mathrm{MG})$ were measured by standard methods and used to calculate the urine albumin-to- $\mathrm{Cr}$ ratio (urinary albumin excretion (UAE)), urine $\alpha 1 \mathrm{MG}$-to-Cr ratio, and urine 5MedC-to-Cr ratio.

2.3. Data Collection. Each subject's age, gender, cause of CKD, complication of diabetes mellitus, medication with antihypertensive drugs (angiotensin receptor blocker (ARB), angiotensin-converting enzyme inhibitor (ACEI), calcium channel blocker (CCB)), mean blood pressure (MBP), and other clinical laboratory data were collected. The serum creatinine concentration was measured by the enzymatic colorimetric method using an automated analyzer (JCA-BM8040; JEOL, Tokyo, Japan), as described previously [19].

2.4. Evaluation of Outcome. The primary outcome was CKD progression, defined as a composite endpoint of incident end-stage renal disease (recipient of maintenance dialysis or renal transplant) or a $30 \%$ decline in the eGFR [22, 23]. Patients were prospectively followed for a median of 36 months (interquartile range, 26-37 months). They were followed by a review of the medical record at least twice a year until December 31, 2014. Loss to follow-up and death were considered censoring events, as described previously [19].

2.5. Statistical Analyses. All values were indicated as the median (interquartile range) or number (percentage). Differences between groups were compared using Wilcoxon's test, a $t$-test, or log-rank test. Kaplan-Meier analyses were applied to assess the effect of urine $5 \mathrm{MedC}$ levels on the renal endpoint using a generalized Wilcoxon test [19, 21]. A multiple regression analysis was used to evaluate the predictors of a low eGFR using the odds ratio (OR) or adjusted OR after adjusting for relevant factors $[18,19,21]$. A $P$ value 
TABLE 1: Baseline characteristics of the study subjects divided by CKD stages.

\begin{tabular}{|c|c|c|c|c|}
\hline & All patients & Early to Mid-CKD (stages 1 to 3 ) & Later CKD (stages 4 and 5) & $P$ value \\
\hline $\mathrm{N}$ & 308 & 241 & 67 & \\
\hline Age (years) & $56(37-67)$ & $52(35-65)$ & $62(55-71)$ & $<0.0001$ \\
\hline Gender, male, n (\%) & $164(53.2)$ & $123(51.0)$ & $41(61.2)$ & 0.139 \\
\hline eGFR $\left(\mathrm{ml} / \mathrm{min} / 1.73 \mathrm{~m}^{2}\right)$ & $55.4(32.0-79.6)$ & $63.8(47.9-85.8)$ & $18.6(14.2-24.3)$ & $<0.0001$ \\
\hline $\mathrm{UAE}(\mathrm{mg} / \mathrm{gCr})$ & $158(20-762)$ & $89(12-542)$ & $705(126-1431)$ & $<0.0001$ \\
\hline $\mathrm{u} \alpha 1 \mathrm{MG}(\mathrm{mg} / \mathrm{gCr})$ & $5.7(2.1-14.1)$ & $3.9(1.7-8.5)$ & $23.5(11.8-48.7)$ & $<0.0001$ \\
\hline u5MedC $(\mu \mathrm{mol} / \mathrm{gCr})$ & $65.9(40.8-130.3)$ & $59.7(39.0-116.5)$ & $88.3(48.5-153.9)$ & 0.025 \\
\hline Hemoglobin (g/L) & $130(116-142)$ & $133(123-146)$ & $112(101-129)$ & $<0.0001$ \\
\hline $\mathrm{MBP}(\mathrm{mmHg})$ & $91(84-99)$ & $91(83-99)$ & $96(85-103)$ & 0.013 \\
\hline Cause of CKD, n (\%) & & & & $<0.0001$ \\
\hline Chronic GN* & $157(51.0)$ & $146(60.6)$ & $11(16.4)$ & \\
\hline Nephrosclerosis & $40(13.0)$ & $19(7.9)$ & $21(31.3)$ & \\
\hline Diabetic nephropathy & $11(3.6)$ & $7(2.9)$ & $4(6.0)$ & \\
\hline Others $* *$ & $100(32.5)$ & $69(28.6)$ & $31(46.3)$ & \\
\hline Diabetes mellitus, n (\%) & $36(11.7)$ & $27(11.2)$ & $9(13.4)$ & 0.615 \\
\hline \multicolumn{5}{|l|}{ Current medication, n (\%) } \\
\hline ARBs/ACEIs & $196(63.0)$ & $137(56.9)$ & $59(88.1)$ & $<0.0001$ \\
\hline CCBs & $117(38.0)$ & $72(29.9)$ & $45(67.2)$ & $<0.0001$ \\
\hline
\end{tabular}

Data are expressed as the median (interquartile) or number (percentage). $\alpha 1 \mathrm{MG}$, alpha1-microglobulin; ARB, angiotensin receptor blocker; ACEI, angiotensinconverting enzyme inhibitor; $\mathrm{CCB}$, calcium channel blocker; $\mathrm{CKD}$, chronic kidney disease; eGFR, estimated glomerular filtration rate; GN, glomerulonephritis; MBP, mean blood pressure; UAE, urinary albumin excretion; u $\alpha 1-\mathrm{MG}$, urinary $\alpha 1$-microglobulin; u5MedC, urinary 5-methyl-2'-deoxycytidine. *Chronic glomerulonephritis includes 93 cases (59.2\%) of IgA nephropathy, 22 cases (14.0\%) of minimal change nephrotic syndrome, 12 cases (7.6\%) of membranous nephropathy, 12 cases (7.6\%) of IgA vasculitis with nephritis, 7 cases (4.5\%) of focal segmental glomerulosclerosis, 6 cases (3.8\%) of non-IgA mesangial nephritis, 4 cases (2.6\%) of membranoproliferative glomerulonephritis and 1 case (0.6\%) of acute glomerulonephritis (persistent and chronic phase). $* *$ Others include 62 cases $(62.0 \%)$ of unknown etiology without a renal biopsy; 20 cases (20.0\%) of lupus nephritis; 10 cases (10.0\%) of antineutrophil cytoplasmic antibody-associated vasculitis; 3 cases (3.0\%) of polycystic kidney disease; 2 cases (2.0\%) of Alport syndrome; and 1 case each (1.0\%) of thin basement membrane disease, cholesterol crystal embolization, and vesicoureteral reflux.

of $<0.05$ was considered to be statistically significant. The SPSS version 20 software package (SPSS Inc., Chicago, IL, USA) and JMP version 11 program (SAS Institute Inc., Cary, NC, USA) were utilized to perform the statistical analyses.

\section{Results}

3.1. Urine $5 \mathrm{MedC}$ Levels and CKD Stages. The baseline profiles of the study subjects are summarized in accordance with the early to middle (stages 1 to 3 ) and later (stages 4 and 5) stages of CKD (Table 1). This study included 308 patients (male, $n=164$; female, $n=144$ ) with a median age of 56 (37-67) years. The background cause of CKD in more than half of the cases was chronic glomerulonephritis (51.0\%). This distribution of patients with chronic glomerulonephritis was similar to that in other nephrology divisions reported in the Japan Renal Biopsy Registry [24]. Significant increases in the levels of albuminuria, urine $\alpha 1 \mathrm{MG}$, and $\mathrm{MBP}$ as well as significant decreases in hemoglobin were recognized, resembling those reported in other cohorts of CKD $[6,25,26]$. The median values of urine $5 \mathrm{MedC}$ were 59.7 and $88.3 \mu \mathrm{mol} / \mathrm{gCr}$ in the early to middle and later CKD stages, respectively (Figure 1). The concentrations of urine $5 \mathrm{MedC}$ were significantly increased in later stages of CKD, suggesting its association with disease progression (Figure 1).
3.2. A Multivariate Analysis to Determine a Low eGFR (Less than $30 \mathrm{~mL} / \mathrm{min} / 1.73 \mathrm{~m}^{2}$ ) in Patients with CKD. Next, separate multiple logistic regression analyses to determine a low eGFR $\left(<30 \mathrm{~mL} / \mathrm{min} / 1.73 \mathrm{~m}^{2}\right)$, which is equivalent to advanced CKD stages 4 and 5, were performed (Table 2). The urine $5 \mathrm{MedC}$ level alone was elucidated to be a significantly independent predictor of a low eGFR (model 1). After adjusting for gender and age as confounding parameters, the urine $5 \mathrm{MedC}$ level was still significant in model 2, which included albuminuria, and model 3, which further included $\mathrm{u} \alpha 1 \mathrm{MG}$ (Table 2). In the univariate analysis, there were no significant correlations between urine 5MedC and other parameters (Table S1). The urine 5MedC levels did not significantly differ when categorized according to the age, gender, cause of CKD, or complications (Table S2).

3.3. Urine $5 \mathrm{MedC}$ in Combination with Other Urine Proteins Significantly Predicts the Renal Survival. During the 36 months of follow-up, 46 patients exhibited a $30 \%$ decline in the eGFR $(n=24)$ or developed end-stage renal disease requiring renal replacement therapy $(n=22)$. There was a higher incidence of disease progression in patients with advanced CKD (stages 4 to 5) (33 of 67 patients) than in those with early to middle CKD (stages 1 to 3 ) (13 of 241 patients). The baseline levels of albuminuria (<300 mg/gCr or $\geq 300 \mathrm{mg} / \mathrm{gCr}$ ) or urine $\alpha 1 \mathrm{MG}$ (median 


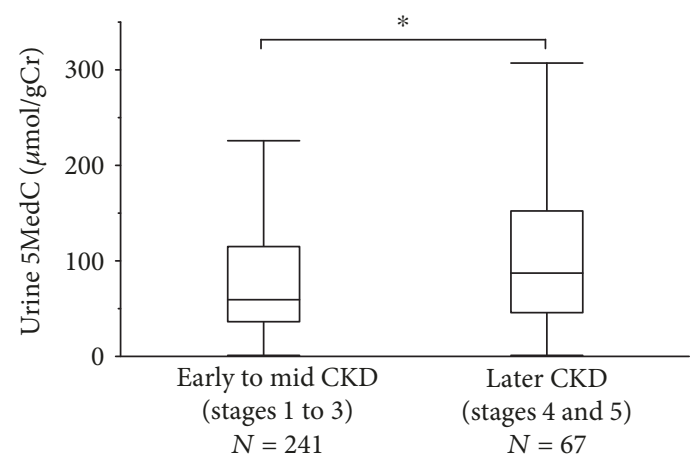

FIgURE 1: Urine 5MedC and CKD stages. Box and line plots showing the levels of urine $5 \mathrm{MedC}(\mu \mathrm{mol} / \mathrm{gCr})$ according to the CKD stages (early to middle stages 1 to 3 or later stages 4 and 5) based on the estimated glomerular filtration rate. The boxes denote the medians and 25th and 75th percentiles. The lines mark the 5th and 95th percentiles. Wilcoxon's test. CKD: chronic kidney disease; 5MedC: 5-methyl-2' -deoxycytidine.

value, $5.7 \mathrm{mg} / \mathrm{gCr}$ ) were able to predict the renal endpointfree survival (Figure S1), suggesting that the CKD cohort in this study was consistent with the relative risk prediction model of CKD $[6,25,26]$. Several studies have investigated the combination of biomarkers to better predict the renal prognosis $[19,26]$. We further performed survival analyses using the level of urine $5 \mathrm{MedC}$ (median value, $65.9 \mu \mathrm{mol} / \mathrm{gCr}$ ) in combination with the level of urine albumin $(<300 \mathrm{mg} / \mathrm{gCr}$ or $\geq 300 \mathrm{mg} / \mathrm{gCr}$ ) or with that of urine $\alpha 1 \mathrm{MG}$ (median value, $5.7 \mathrm{mg} / \mathrm{gCr}$ ) in Kaplan-Meier survival curves (Figure 2). An increased urine $5 \mathrm{MedC}$ level in CKD patients did not significantly predict a worse renal outcome than a lower urine 5MedC level (Figure S1); however, a significant effect of an increased urine $5 \mathrm{MedC}$ level on predicting a poor renal survival when combined with macroalbuminuria $(\geq 300 \mathrm{mg} / \mathrm{gCr})$ or an increased urine $\alpha 1 \mathrm{MG}$ level was observed (Figure 2).

3.4. Relationship between Urine 5MedC, a Marker of DNA Methylation, and Urine 8-OHdG, a Marker of Oxidized $D N A$. We carried out a further analysis of the 273 patients with available data for urine 8-OHdG, a marker of oxidized DNA due to oxidative stress. We recognized a significant univariate correlation between $5 \mathrm{MedC}$ and $8-\mathrm{OHdG}$ in the urine of CKD patients, suggesting an association between DNA methylation and oxidized DNA and thus a linkage between epigenetic and genetic alterations in such patients (Figure 3).

\section{Discussion}

In the genomic DNA of mammals, methylation of the C-5 position of cytosine is a key mechanism of epigenetic control that influences the gene expression, stability of genome, and differentiation of cells [27]. Abnormal methylation of several genes, either hypermethylation or hypomethylation, has been involved in various diseases, including cancer [2, 28, 29], diabetes [30], obesity [31], Alzheimer's disease [32], and schizophrenia [5]. The level of 5-methylcytidine $(5 \mathrm{MeC})$ is determined by the balance between DNA methylation and
DNA demethylation processes. DNA methylation is catalyzed by DNA methyltransferases, with S-adenosylmethionine functioning as a donor of methyl. DNA methylation may be removed enzymatically by certain mechanisms including BER [33], NER, and hydrolysis [34-36].

In this study, we measured the urine level of $5 \mathrm{MedC}$, a marker of repair products of DNA methylation, in patients with $\mathrm{CKD}$ and investigated the relationships between the urine 5MedC level and CKD progression and outcomes. Herein, we provide the evidence that (1) the urine $5 \mathrm{MedC}$ level was significantly increased in the later stages of CKD (i.e., eGFR less than $30 \mathrm{~mL} / \mathrm{min} / 1.73 \mathrm{~m}^{2}$ ) and was associated with a later CKD stage according to a multiple regression analysis even after adjusting for confounding parameters; furthermore, (2) while urine $5 \mathrm{MedC}$ alone did not significantly predict the renal outcome in CKD patients, a significant effect of urine $5 \mathrm{MedC}$ on predicting a poor renal outcome when combined with macroalbuminuria or an increased urine $\alpha 1 \mathrm{MG}$ level was detected.

$5 \mathrm{MedC}$ is a product of the BER and NER pathways of active DNA methylation. DNA repair products, including 5MedC, 5-hydroxymethylcytosine, 5-formylcytosine, and 5carboxycytosine, are released into the blood and subsequently appear in the urine $[37,38]$. Several techniques have been developed for the determination of $5 \mathrm{MedC}$ in human urine samples, such as immunochemical detection [13], ion-pair liquid chromatography (LC) [16], LC with mass spectrometry (LC-MS) [14], LC with tandem mass spectrometry (LC-MS/MS) [12], and high-performance LC with tandem mass spectrometry (HPLC-MS/MS) $[15,39]$.

Itoh et al. reported that an ELISA with specific monoclonal antibodies was able to detect $5 \mathrm{MedC}$ as the major immunoreactive nucleoside in the urine of a healthy human and increased concentrations of urine $5 \mathrm{MedC}$ were observed in leukemic patients with active diseases [13]. The mean levels of urine $5 \mathrm{MedC}$ in healthy subjects were $0.90 \pm 0.43 \mathrm{nmol} / \mu$ molCr in that analysis. The generation of $5 \mathrm{MedC}$ may be caused by the active excision repair of DNA in human cells. Heavily methylated DNA of leukemic cells may be the origin of increased $5 \mathrm{MedC}$ in the urine of leukemic patients. Zambonin et al. then applied a simple reversed-phase LC technique to determine the urine $5 \mathrm{MedC}$ levels normalized by urine creatinine excretion in healthy individuals and patients with leukemia [16]. Lee et al. further analyzed the urine levels of oxidized nucleosides using LC with electrospray mass spectrometry and found that the urine $5 \mathrm{MedC}$ levels did not significantly change, but those of 8-OHdG were significantly elevated in patients with Alzheimer's disease compared to healthy subjects [14]. The mean urine level of $5 \mathrm{MedC}$ was $0.262 \pm 0.156 \mathrm{nmol} / \mu \mathrm{molCr}$ in that study. Based on these previous findings, we initially excluded patients with cancer and Alzheimer's disease from the present study.

$\mathrm{Hu}$ et al. measured the level of urine $5 \mathrm{MeC}$ and $5 \mathrm{MedC}$ by LC-MS/MS with isotope dilution in healthy males and found that the concentration of urine $5 \mathrm{MeC}$ was significantly correlated with those of methylated purines and lesions of oxidized DNA, including 8-oxo-7,8-dihydro- $2^{\prime}$-deoxyguanosine (8-oxodG) [12]. The mean urine level of $5 \mathrm{MedC}$ was $7.04 \pm 7.2 \mathrm{ng} / \mathrm{mgCr}$ in that report. The level of urine $5 \mathrm{MedC}$, 
TABLe 2: A multiple logistic regression analysis to determine low eGFR (later CKD stages, $<30 \mathrm{~mL} / \mathrm{min} / 1.73 \mathrm{~m}^{2}$ ) in different models.

\begin{tabular}{|c|c|c|c|}
\hline & Odds ratio & $95 \% \mathrm{CI}$ & $P$ value \\
\hline \multicolumn{4}{|l|}{ Model 1} \\
\hline $\mathrm{u} 5 \mathrm{MedC} \geq$ median $(\mu \mathrm{mol} / \mathrm{gCr})$ & 2.30 & $1.29-4.21$ & 0.005 \\
\hline \multicolumn{4}{|l|}{ Model 2} \\
\hline $\mathrm{u} 5 \mathrm{MedC} \geq \operatorname{median}(\mu \mathrm{mol} / \mathrm{gCr})$ & 2.16 & $1.18-4.04$ & 0.012 \\
\hline $\mathrm{UAE} \geq 300(\mathrm{mg} / \mathrm{gCr})$ & 4.31 & $2.36-8.08$ & $<0.0001$ \\
\hline \multicolumn{4}{|l|}{ Model 3} \\
\hline $\mathrm{u} 5 \mathrm{MedC} \geq \operatorname{median}(\mu \mathrm{mol} / \mathrm{gCr})$ & 2.36 & $1.24-4.60$ & 0.008 \\
\hline $\mathrm{UAE} \geq 300(\mathrm{mg} / \mathrm{gCr})$ & 1.39 & $0.67-2.90$ & 0.381 \\
\hline $\mathrm{u} \alpha 1 \mathrm{MG} \geq \operatorname{median}(\mathrm{mg} / \mathrm{gCr})$ & 13.56 & $5.32-40.1$ & $<0.0001$ \\
\hline
\end{tabular}

Adjusted for age and gender. The median values of u5MedC and $\mathrm{u} \alpha 1 \mathrm{MG}$ are $65.9(\mu \mathrm{mol} / \mathrm{gCr})$ and 5.7 (mg/gCr), respectively. CKD, chronic kidney disease; eGFR, estimated glomerular filtration rate; UAE, urinary albumin excretion; u $\alpha 1$-MG, urinary $\alpha 1$-microglobulin; u5MedC, urinary 5-methyl-2'deoxycytidine; $\mathrm{CI}$, confidence interval.

however, did not correlate with any methylated or oxidized lesions in healthy male subjects in that study. Pan et al. investigated the levels of urine 5MedC and 5-hydroxymethyl-2' -deoxycytidine (5hMedC) by HPLC-MS/MS in subfertile men and showed their associations with phthalate metabolites (environmental chemicals) and semen parameters (healthy outcomes), suggesting that these are promising biomarkers for use in epidemiological studies $[15,39]$. In addition to urine samples of humans, other researchers have attempted to determine the 5MedC level in DNA obtained from human peripheral blood [40] or human lung cancer tissue [41] by LC-MS/MS as well as in DNA obtained from cultured Hela cells by HPLC-ultraviolet detection [42] and from newborn cord blood samples by HPLC-MS/MS [43].

Recent reports have identified roles of environmental [6], genetic $[44,45]$, and epigenetic factors $[46,47]$ in the progression of CKD. Epigenetic risk factors for CKD have only recently been investigated [10], and the DNA methylation profile in the blood might be associated with a rapid decline in the renal function [48]. In the present study, the group exhibiting both higher levels of urine $5 \mathrm{MedC}$ and albuminuria had a worse renal survival than the group exhibiting lower levels of both (Figure 2). Whether albuminuria induces epigenetic changes, including DNA methylation, and thus an increase in urine $5 \mathrm{MedC}$ in resident kidney cells is largely unknown. The expression of Krüppel-like factor 4, which can reprogram somatic cells into induced pluripotent stem cells, reduced DNA methylation at the nephrin promoter, which may lead to protection against albuminuria [49]. The hypomethylation of aldo-keto reductase family 1 member B1 and tissue inhibitor of metalloproteinase 2 genes in association with albuminuria has been reported in subjects with early stages of diabetic nephropathy [50], although we did not recognize a significant correlation between urine $5 \mathrm{MedC}$ and albuminuria levels in the univariate analysis in our cohort (Table S1).

We found in the present study that urine 5MedC levels were significantly increased in the later stages of CKD (stages 4 and 5, i.e., eGFR less than $30 \mathrm{~mL} / \mathrm{min} / 1.73 \mathrm{~m}^{2}$ ) (Figure 1), when uremic toxins may be detected in both the urine and serum of such patients. In recent reports, uremia was shown to induce alterations in DNA methylation in differentiating monocytes in patients with CKD [51]. The expression of the antiaging and renoprotective gene klotho is known to be suppressed under conditions of uremia [18]. The protein-bound uremic toxins can increase the DNA methyltransferase and DNA methylation, thereby leading to the suppression of the klotho expression in the uremic milieu [52]. Therefore, certain uremic toxins might alter the global DNA methylation and the expression of urine 5MedC in CKD patients. In rodent models, hypermethylation of certain genes is involved in the activation of fibroblasts and fibrogenesis in the kidney, which may be one of the molecular mechanisms associated with the progression of CKD [53].

Epigenetic patterns can change over one's lifetime, suggesting that epigenetic changes may constitute an important factor of the aging process [54]. Since CKD might be an aging-related disorder, we investigated the urine $5 \mathrm{MedC}$ level in different age categories in our CKD cohort. However, the CKD patients $\geq 75$ years of age did not exhibit a significantly different level of urine $5 \mathrm{MedC}$ than those $<75$ years of age in our study (Table S2). We recognized the correlation between urine 5MedC, a marker of global DNA methylation, and urine $8-\mathrm{OHdG}$, a marker of oxidized DNA by oxidative stress (Figure 3), suggesting a link between DNA oxidation and DNA methylation. There might therefore be a connection between genetic and epigenetic alterations, possibly via oxidative stress in such patients. Several reports have investigated the relationship between oxidized DNA and DNA methylation [55-58], including the simultaneous examination of $8-\mathrm{OHdG}$ and $5 \mathrm{MedC}$ in DNA samples [55]. 8-OHdG may induce hypomethylation of DNA by inhibiting DNA methylation at nearby cytosine bases [58]. Significantly negative correlations were reported between 8OHdG and levels of global methylation in DNA extracted from leukocytes in workers exposed to nanomaterials of metal oxide [56] and between plasma 8-OHdG and global methylation levels in leukocyte DNA in subjects with biliary atresia [57]. Further investigations are thus required in order to clarify the association between oxidized DNA and DNA methylation.

This study has several limitations and strengths that must be kept in mind when understanding the data. First, urine $5 \mathrm{MedC}$ did not exhibit methylation of specific genes involved 


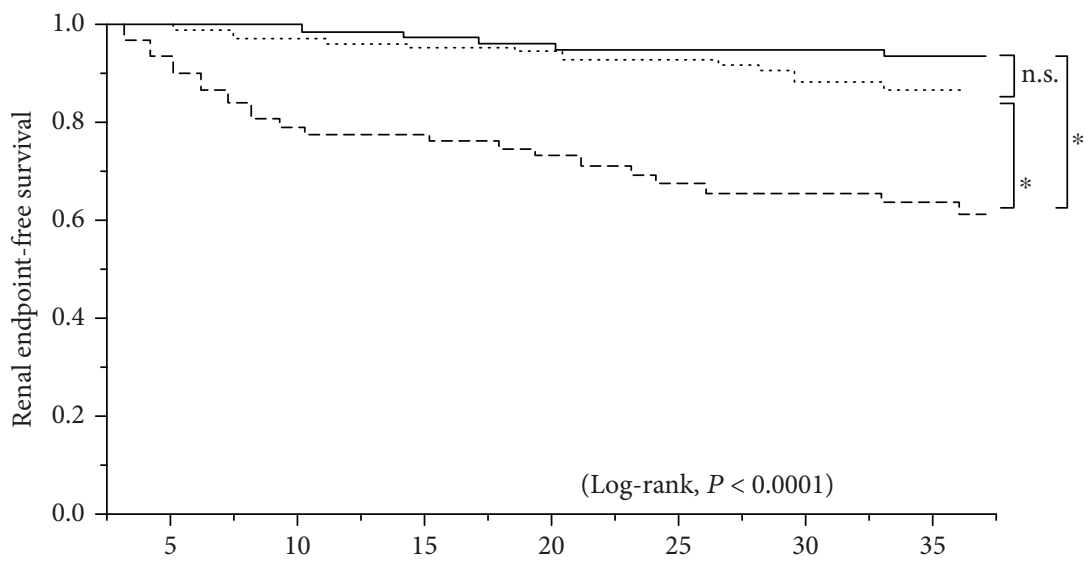

No. at risk

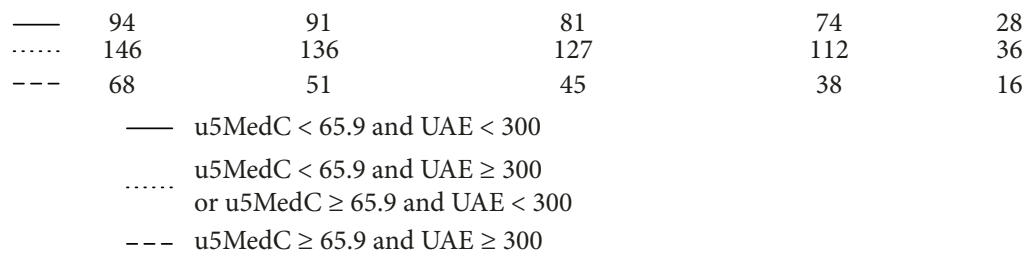

(a)

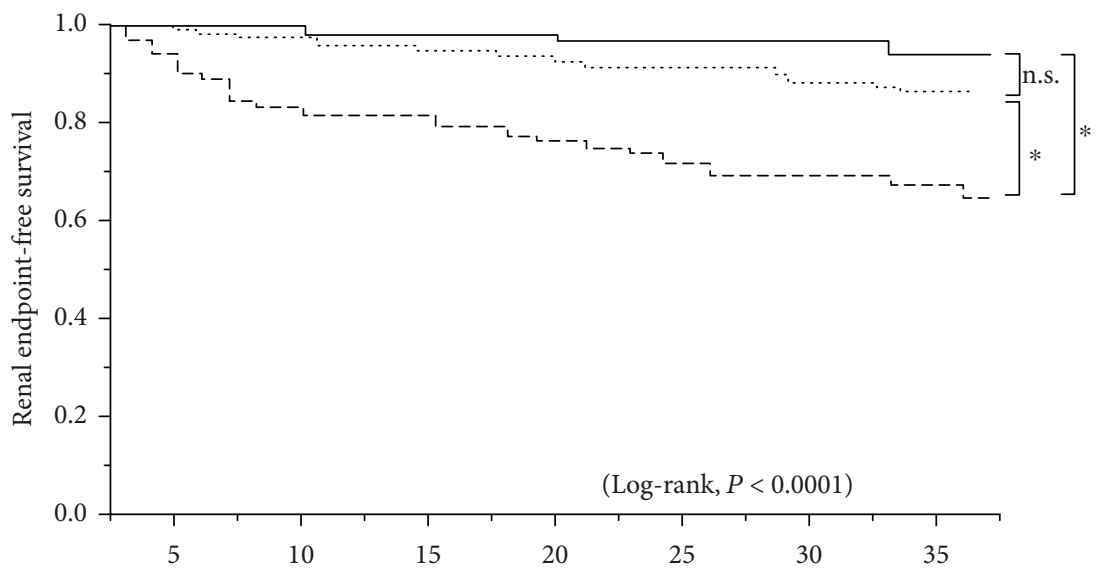

No. at risk

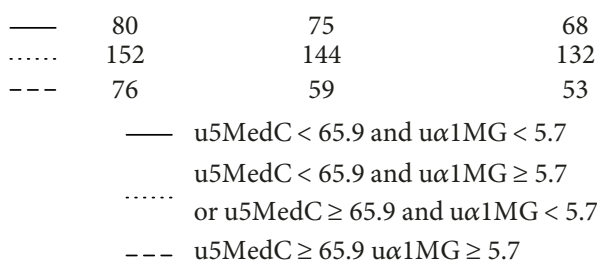

(b)

Figure 2: Urine 5MedC and CKD outcome. Kaplan-Meier curves showing the renal endpoint-free survival categorized by urine 5MedC $(\mu \mathrm{mol} / \mathrm{gCr})$ and its combination with albuminuria $(\mathrm{mg} / \mathrm{gCr})$ (a) or urine $\alpha 1 \mathrm{MG}(\mathrm{mg} / \mathrm{gCr})(\mathrm{b})$. The combination of urine $5 \mathrm{MedC}$ with albuminuria (a) or urine $\alpha 1 \mathrm{MG}$ (b) clearly separated the three-year renal endpoint-free survival of CKD patients. (a) u5MedC $<65.9$ and $\mathrm{UAE}<300, n=94(30.5 \%)$; u5MedC $<65.9$ and $\mathrm{UAE} \geq 300$ or u5MedC $\geq 65.9$ and $\mathrm{UAE}<300, n=146(47.4 \%)$; and $\mathrm{u} 5 \mathrm{MedC} \geq 65.9$ and $\mathrm{UAE} \geq 300, n=68(22.1 \%)$. (b) u5MedC $<65.9$ and $\mathrm{u} \alpha 1 \mathrm{MG}<5.7, n=80(26.0 \%)$; u $5 \mathrm{MedC}<65.9$ and $\mathrm{u} \alpha 1 \mathrm{MG} \geq 5.7$ or $\mathrm{u} 5 \mathrm{MedC} \geq 65.9$ and $\mathrm{u} \alpha 1 \mathrm{MG}<5.7, n=152(49.4 \%)$; $\mathrm{u} 5 \mathrm{MedC} \geq 65.9$ and $\mathrm{u} \alpha 1 \mathrm{MG} \geq 5.7, n=76(24.7 \%)$. * indicates $P<0.0001$, n.s. denotes not significant. Log-rank test. UAE: urinary albumin excretion; u $\alpha 1 \mathrm{MG}$ : urinary alpha1-microglobulin; u5MedC: urinary 5-methyl-2' ${ }^{\prime}$-deoxycytidine.

in CKD, such as polycystic kidney disease 1 [59] but exhibited global DNA methylation. Second, while three major enzymes are necessary for de novo DNA methylation (DNMT3A and
DNMT3B) or maintenance methylation (DNMT1) in mammalian cells [3], we did not examine the levels of these enzymes in the present study. Third, we did not have 


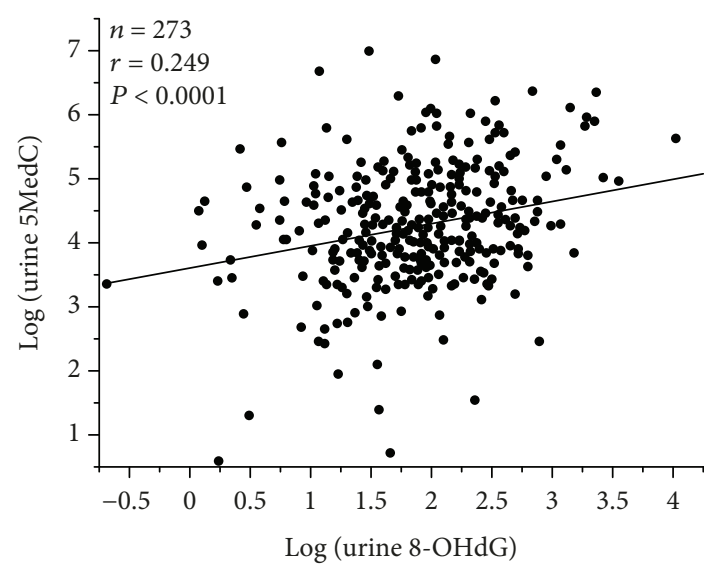

FIGURE 3: Relationship between levels of urine $5 \mathrm{MedC}$ and urine 8OHdG. The level of urine $5 \mathrm{MedC}(\mu \mathrm{mol} / \mathrm{gCr})$ significantly correlates with the level of urine 8 -OHdG $(\mathrm{ng} / \mathrm{mgCr})$ in patients with CKD $(n=273)$. $t$-test. 5MedC: 5 -methyl- $2^{\prime}$-deoxycytidine; 8 OHdG: 8-hydroxy-2'-deoxyguanosine.

sufficient data on subjects with diabetic nephropathy, which is the most frequent cause of ESRD in several countries. However, including diabetic subjects in the CKD cohort may have influenced the $5 \mathrm{MedC}$ levels, as alterations in the DNA methylation of the gene network occur under conditions of diabetes mellitus [60] and in glomerular podocytes under conditions of diabetic nephropathy [61, 62]. Fourth, the serum and kidney tissue levels of $5 \mathrm{MedC}$ were not investigated in this study, as we did not obtain these samples in our setting. Renal compartment-specific genetic and epigenetic analyses would be able to identify further novel mechanisms involved in the progression of $\mathrm{CKD}$ [63]. Fifth, we were unable to evaluate cardiovascular events in CKD patients because we expected a low number of such events in our setting, although epigenetic dysregulation of CKD-associated cardiovascular disease might be relevant [64]. In addition, we were unable to confirm the level of $5 \mathrm{MedC}$ in our urine samples using other methodologies, such as LC-MS/MS [12]. Other caveats include the lack of data on lifestyle risks, such as smoking and toxin exposure, as potential confounders.

\section{Conclusions}

We examined the levels of urine 5MedC, a marker of DNA methylation and an epigenetic marker, in patients with CKD. These values significantly increased in the later CKD stages and were related to a reduced eGFR. The urine $5 \mathrm{MedC}$ level in combination with albuminuria or the $\alpha 1 \mathrm{MG}$ level significantly predicted the renal survival in CKD patients, suggesting that it can serve as a novel biomarker for predicting the renal outcome in CKD, which is a significant issue given the currently increasing number of CKD patients all over the world. Our previous studies and others suggested urine trefoil factors to be biomarkers for progression of CKD [19, 65, 66]; however, recent studies demonstrated these small peptides as biomarkers for acute kidney injury [67] and drug-induced kidney injury [68]. We thus believe urine $5 \mathrm{MedC}$ as a promising and novel biomarker for CKD based on the current study.

Further studies to clarify the kidney disease-specific changes in the level of 5MedC utilizing a larger CKD cohort and exploring the renal compartment-specific epigenetic analyses will be necessary. Clarifying whether intervention and treatment of CKD patients with agents such as cholesterol-lowering medications [69] can alter the level of urine $5 \mathrm{MedC}$ is of great importance.

\section{Data Availability}

No data is used to support this study.

\section{Conflicts of Interest}

Jun Wada takes honoraria as a speaker from Daiichi Sankyo, MSD, Tanabe Mitsubishi, and Taisho Toyama and receives support from a grant from Baxter, Dainippon Sumitomo, Ono, and Teijin Pharma. Haruhito A. Uchida belongs to the Department of Chronic Kidney Disease and Cardiovascular Disease which is supported by Chugai Pharmaceutical, MSD, Boehringer Ingelheim, and Kawanishi Holdings. The other authors declare that they have no competing interests.

\section{Acknowledgments}

We sincerely thank all of the participating patients, collaborating physicians, and other medical staff in our department for their contributions. A part of this work was supported by JSPS KAKENHI Grant Numbers JP16K09616 and 19K08679 to HS.

\section{Supplementary Materials}

Supplementary Table S1: univariate correlation between urinary $5 \mathrm{MedC}$ and other clinical parameters. Supplementary Table S2: urinary 5MedC levels according to age, gender, cause of CKD, and complication of diabetes. Supplementary Figure S1: the renal survival categorized by albuminuria, urine $\alpha 1 \mathrm{MG}$, and urine 5MedC alone. (Supplementary Materials)

\section{References}

[1] A. P. Feinberg and M. D. Fallin, "Epigenetics at the crossroads of genes and the environment," JAMA, vol. 314, no. 11, pp. 1129-1130, 2015.

[2] P. A. Jones and S. B. Baylin, "The epigenomics of cancer," Cell, vol. 128, no. 4, pp. 683-692, 2007.

[3] T. Chen and E. Li, "Structure and function of eukaryotic DNA methyltransferases," Current Topics in Developmental Biology, vol. 60, pp. 55-89, 2004.

[4] K. D. Robertson, "DNA methylation and human disease," Nature Reviews Genetics, vol. 6, no. 8, pp. 597-610, 2005.

[5] C. Montano, M. A. Taub, A. Jaffe et al., "Association of DNA methylation differences with schizophrenia in an epigenomewide association study," JAMA Psychiatry, vol. 73, no. 5, pp. 506-514, 2016. 
[6] A. S. Levey and J. Coresh, "Chronic kidney disease," The Lancet, vol. 379, no. 9811, pp. 165-180, 2012.

[7] K. F. National, "K/DOQI clinical practice guidelines for chronic kidney disease: evaluation, classification, and stratification," American Journal of Kidney Diseases: The Official Journal of the National Kidney Foundation, vol. 39, no. 2, Supplement 1, p. S1, 2002.

[8] B. C. Astor, K. Matsushita, R. T. Gansevoort et al., "Lower estimated glomerular filtration rate and higher albuminuria are associated with mortality and end-stage renal disease. A collaborative meta-analysis of kidney disease population cohorts," Kidney International, vol. 79, no. 12, pp. 13311340, 2011.

[9] R. G. Fassett, S. K. Venuthurupalli, G. C. Gobe, J. S. Coombes, M. A. Cooper, and W. E. Hoy, "Biomarkers in chronic kidney disease: a review," Kidney International, vol. 80, no. 8, pp. 806821, 2011.

[10] L. J. Smyth, G. J. McKay, A. P. Maxwell, and A. J. McKnight, "DNA hypermethylation and DNA hypomethylation is present at different loci in chronic kidney disease," Epigenetics, vol. 9, no. 3, pp. 366-376, 2014

[11] S. Kato, B. Lindholm, P. Stenvinkel et al., "DNA hypermethylation and inflammatory markers in incident Japanese dialysis patients," Nephron Extra, vol. 2, no. 1, pp. 159-168, 2012.

[12] C. W. Hu, H. H. Liu, Y. J. Li, and M. R. Chao, "Direct analysis of 5-methylcytosine and 5-methyl-2' -deoxycytidine in human urine by isotope dilution LC-MS/MS: correlations with $\mathrm{N}$ methylated purines and oxidized DNA lesions," Chemical Research in Toxicology, vol. 25, no. 2, pp. 462-470, 2012.

[13] K. Itoh, S. Aida, S. Ishiwata, T. Yamaguchi, N. Ishida, and M. Mizugaki, "Immunochemical detection of urinary 5methyl-2' -deoxycytidine as a potential biologic marker for leukemia," Clinica Chimica Acta, vol. 234, no. 1-2, pp. 37-45, 1995.

[14] S. H. Lee, I. Kim, and B. C. Chung, "Increased urinary level of oxidized nucleosides in patients with mild-to-moderate Alzheimer's disease," Clinical Biochemistry, vol. 40, no. 13-14, pp. 936-938, 2007.

[15] Y. Pan, J. Jing, L. W. Yeung et al., “Associations of urinary 5-methyl-2'-deoxycytidine and 5-hydroxymethyl-2' -deoxycytidine with phthalate exposure and semen quality in 562 Chinese adult men," Environment International, vol. 94, pp. 583-590, 2016.

[16] C. G. Zambonin, A. Aresta, F. Palmisano, G. Specchia, and V. Liso, "Liquid chromatographic determination of urinary 5-methyl-2' -deoxycytidine and pseudouridine as potential biological markers for leukaemia," Journal of Pharmaceutical and Biomedical Analysis, vol. 21, no. 5, pp. 1045-1051, 1999.

[17] S. Matsuo, E. Imai, M. Horio et al., "Revised equations for estimated GFR from serum creatinine in Japan," American Journal of Kidney Diseases, vol. 53, no. 6, pp. 982-992, 2009.

[18] M. Kitagawa, H. Sugiyama, H. Morinaga et al., "A decreased level of serum soluble klotho is an independent biomarker associated with arterial stiffness in patients with chronic kidney disease," PLoS One, vol. 8, no. 2, article e56695, 2013.

[19] T. Yamanari, H. Sugiyama, K. Tanaka et al., "Urine trefoil factors as prognostic biomarkers in chronic kidney disease," BioMed Research International, vol. 2018, Article ID 3024698, 11 pages, 2018.

[20] N. Fukuoka, H. Sugiyama, T. Inoue et al., "Increased susceptibility to oxidant-mediated tissue injury and peritoneal fibrosis in acatalasemic mice," American Journal of Nephrology, vol. 28, no. 4, pp. 661-668, 2008.

[21] H. Morinaga, H. Sugiyama, T. Inoue et al., "Effluent free radicals are associated with residual renal function and predict technique failure in peritoneal dialysis patients," Peritoneal Dialysis International, vol. 32, no. 4, pp. 453-461, 2012.

[22] J. Coresh, T. C. Turin, K. Matsushita et al., "Decline in estimated glomerular filtration rate and subsequent risk of endstage renal disease and mortality," JAMA, vol. 311, no. 24, pp. 2518-2531, 2014.

[23] E. Kanda, T. Usui, N. Kashihara, C. Iseki, K. Iseki, and $\mathrm{M}$. Nangaku, "Importance of glomerular filtration rate change as surrogate endpoint for the future incidence of end-stage renal disease in general Japanese population: communitybased cohort study," Clinical and Experimental Nephrology, vol. 22, no. 2, pp. 318-327, 2018.

[24] Committee for Standardization of Renal Pathological Diagnosis and Committee for Kidney Disease Registry, Japanese Society of Nephrology, Japan, H. Sugiyama, H. Yokoyama et al., "Japan Renal Biopsy Registry and Japan Kidney Disease Registry: committee report for 2009 and 2010," Clinical and Experimental Nephrology, vol. 17, no. 2, pp. 155-173, 2013.

[25] A. S. Levey, P. E. de Jong, J. Coresh et al., "The definition, classification, and prognosis of chronic kidney disease: a KDIGO controversies conference report," Kidney International, vol. 80, no. 1, pp. 17-28, 2011.

[26] Y. Otaki, T. Watanabe, T. Shishido et al., "The impact of renal tubular damage, as assessed by urinary $\beta_{2}$-microglobulin-creatinine ratio, on cardiac prognosis in patients with chronic heart failure," Circulation: Heart Failure, vol. 6, no. 4, pp. 662-668, 2013.

[27] M. Gehring, W. Reik, and S. Henikoff, "DNA demethylation by DNA repair," Trends in Genetics, vol. 25, no. 2, pp. 82-90, 2009.

[28] M. Hatziapostolou and D. Iliopoulos, "Epigenetic aberrations during oncogenesis," Cellular and Molecular Life Sciences, vol. 68, no. 10, pp. 1681-1702, 2011.

[29] B. Thienpont, J. Steinbacher, H. Zhao et al., "Tumour hypoxia causes DNA hypermethylation by reducing TET activity," Nature, vol. 537, no. 7618, pp. 63-68, 2016.

[30] L. Sommese, A. Zullo, F. P. Mancini, R. Fabbricini, A. Soricelli, and C. Napoli, "Clinical relevance of epigenetics in the onset and management of type 2 diabetes mellitus," Epigenetics, vol. 12, no. 6, pp. 401-415, 2017.

[31] M. M. Mendelson, R. E. Marioni, R. Joehanes et al., "Association of body mass index with DNA methylation and gene expression in blood cells and relations to cardiometabolic disease: a Mendelian randomization approach," PLoS Medicine, vol. 14, no. 1, article e1002215, 2017.

[32] C. T. Watson, P. Roussos, P. Garg et al., "Genome-wide DNA methylation profiling in the superior temporal gyrus reveals epigenetic signatures associated with Alzheimer's disease," Genome Medicine, vol. 8, no. 1, p. 5, 2016.

[33] M. Vairapandi and N. J. Duker, "Enzymic removal of 5methylcytosine from DNA by a human DNA-glycosylase," Nucleic Acids Research, vol. 21, no. 23, pp. 5323-5327, 1993.

[34] G. Barreto, A. Schäfer, J. Marhold et al., "Gadd45a promotes epigenetic gene activation by repair-mediated DNA demethylation," Nature, vol. 445, no. 7128, pp. 671-675, 2007.

[35] S. K. Bhattacharya, S. Ramchandani, N. Cervoni, and M. Szyf, "A mammalian protein with specific demethylase 
activity for mCpG DNA," Nature, vol. 397, no. 6720, pp. 579-583, 1999.

[36] J. K. Zhu, "Active DNA demethylation mediated by DNA glycosylases," Annual Review of Genetics, vol. 43, no. 1, pp. 143-166, 2009.

[37] S. Kriaucionis and N. Heintz, "The nuclear DNA base 5hydroxymethylcytosine is present in Purkinje neurons and the brain," Science, vol. 324, no. 5929, pp. 929-930, 2009.

[38] M. Tahiliani, K. P. Koh, Y. Shen et al., "Conversion of 5methylcytosine to 5-hydroxymethylcytosine in mammalian DNA by MLL partner TET1," Science, vol. 324, no. 5929, pp. 930-935, 2009.

[39] R. Yin, J. Mo, M. Lu, and H. Wang, "Detection of human urinary 5-hydroxymethylcytosine by stable isotope dilution HPLC-MS/MS analysis," Analytical Chemistry, vol. 87, no. 3, pp. 1846-1852, 2015.

[40] X. Li and A. A. Franke, "High-throughput and cost-effective global DNA methylation assay by liquid chromatographymass spectrometry," Analytica Chimica Acta, vol. 703, no. 1, pp. 58-63, 2011.

[41] C.-W. Hu, H. Lee, J.-L. Chen, Y.-J. Li, and M.-R. Chao, “Optimization of global DNA methylation measurement by LCMS/MS and its application in lung cancer patients," Analytical and Bioanalytical Chemistry, vol. 405, no. 27, pp. 8859-8869, 2013.

[42] J. Sandhu, B. Kaur, C. Armstrong et al., "Determination of 5-methyl-2' -deoxycytidine in genomic DNA using high performance liquid chromatography-ultraviolet detection," Journal of Chromatography. B, Analytical Technologies in the Biomedical and Life Sciences, vol. 877, no. 20-21, pp. 19571961, 2009.

[43] P. Intarasunanont, P. Navasumrit, S. Waraprasit et al., "Effects of arsenic exposure on DNA methylation in cord blood samples from newborn babies and in a human lymphoblast cell line," Environmental Health, vol. 11, no. 1, 2012.

[44] J. J. Grantham, V. E. Torres, A. B. Chapman et al., "Volume progression in polycystic kidney disease," New England Journal of Medicine, vol. 354, no. 20, pp. 2122-2130, 2006.

[45] A. Parsa, W. H. Kao, D. Xie et al., "APOL1 risk variants, race, and progression of chronic kidney disease," The New England Journal of Medicine, vol. 369, no. 23, pp. 21832196, 2013.

[46] P. Stenvinkel, M. Karimi, S. Johansson et al., "Impact of inflammation on epigenetic DNA methylation - a novel risk factor for cardiovascular disease?," Journal of Internal Medicine, vol. 261, no. 5, pp. 488-499, 2007.

[47] K. Susztak, "Understanding the epigenetic syntax for the genetic alphabet in the kidney," Journal of the American Society of Nephrology, vol. 25, no. 1, pp. 10-17, 2014.

[48] M. R. Wing, J. M. Devaney, M. M. Joffe et al., "DNA methylation profile associated with rapid decline in kidney function: findings from the CRIC study," Nephrology, Dialysis, Transplantation, vol. 29, no. 4, pp. 864-872, 2014.

[49] K. Hayashi, H. Sasamura, M. Nakamura et al., "KLF4-dependent epigenetic remodeling modulates podocyte phenotypes and attenuates proteinuria," The Journal of Clinical Investigation, vol. 124, no. 6, pp. 2523-2537, 2014.

[50] O. Aldemir, F. Turgut, and C. Gokce, "The association between methylation levels of targeted genes and albuminuria in patients with early diabetic kidney disease," Renal Failure, vol. 39, no. 1, pp. 597-601, 2017.
[51] A. M. Zawada, J. S. Schneider, A. I. Michel et al., "DNA methylation profiling reveals differences in the 3 human monocyte subsets and identifies uremia to induce DNA methylation changes during differentiation," Epigenetics, vol. 11, no. 4, pp. 259-272, 2016.

[52] C. Y. Sun, S. C. Chang, and M. S. Wu, "Suppression of klotho expression by protein-bound uremic toxins is associated with increased DNA methyltransferase expression and DNA hypermethylation," Kidney International, vol. 81, no. 7, pp. 640-650, 2012.

[53] W. Bechtel, S. McGoohan, E. M. Zeisberg et al., "Methylation determines fibroblast activation and fibrogenesis in the kidney," Nature Medicine, vol. 16, no. 5, pp. 544-550, 2010.

[54] M. J. Jones, S. J. Goodman, and M. S. Kobor, "DNA methylation and healthy human aging," Aging Cell, vol. 14, no. 6, pp. 924-932, 2015.

[55] J. Hu, W. Zhang, H. Ma, Y. Cai, G. Sheng, and J. Fu, "Simultaneous determination of 8-hydroxy- $2^{\prime}$-deoxyguanosine and 5methyl-2' -deoxycytidine in DNA sample by high performance liquid chromatography/positive electrospray ionization tandem mass spectrometry," Journal of Chromatography. B, Analytical Technologies in the Biomedical and Life Sciences, vol. 878, no. 28, pp. 2765-2769, 2010.

[56] S. H. Liou, W. T. Wu, H. Y. Liao et al., "Global DNA methylation and oxidative stress biomarkers in workers exposed to metal oxide nanoparticles," Journal of Hazardous Materials, vol. 331, pp. 329-335, 2017.

[57] W. Udomsinprasert, N. Kitkumthorn, A. Mutirangura, V. Chongsrisawat, Y. Poovorawan, and S. Honsawek, "Global methylation, oxidative stress, and relative telomere length in biliary atresia patients," Scientific Reports, vol. 6, no. 1, article 26969, 2016.

[58] Q. Wu and X. Ni, "ROS-mediated DNA methylation pattern alterations in carcinogenesis," Current Drug Targets, vol. 16, no. 1, pp. 13-19, 2015.

[59] Y. M. Woo, J. B. Bae, Y. H. Oh et al., "Genome-wide methylation profiling of ADPKD identified epigenetically regulated genes associated with renal cyst development," Human Genetics, vol. 133, no. 3, pp. 281-297, 2014.

[60] J. Kang, C. N. Lee, H. Y. Li, K. H. Hsu, and S. Y. Lin, "Genomewide DNA methylation variation in maternal and cord blood of gestational diabetes population," Diabetes Research and Clinical Practice, vol. 132, pp. 127-136, 2017.

[61] K. Hasegawa, S. Wakino, P. Simic et al., "Renal tubular Sirt1 attenuates diabetic albuminuria by epigenetically suppressing claudin-1 overexpression in podocytes," Nature Medicine, vol. 19, no. 11, pp. 1496-1504, 2013.

[62] T. Marumo, S. Yagi, W. Kawarazaki et al., "Diabetes induces aberrant DNA methylation in the proximal tubules of the kidney," Journal of the American Society of Nephrology, vol. 26, no. 10, pp. 2388-2397, 2015.

[63] C. Qiu, S. Huang, J. Park et al., "Renal compartment-specific genetic variation analyses identify new pathways in chronic kidney disease," Nature Medicine, vol. 24, no. 11, pp. 17211731, 2018.

[64] A. M. Zawada, K. S. Rogacev, and G. H. Heine, "Clinical relevance of epigenetic dysregulation in chronic kidney disease-associated cardiovascular disease," Nephrology Dialysis Transplantation, vol. 28, no. 7, pp. 1663-1671, 2013. 
[65] D. Lebherz-Eichinger, B. Tudor, H. J. Ankersmit et al., "Increased trefoil factor 2 levels in patients with chronic kidney disease," PLoS One, vol. 12, no. 3, article e0174551, 2017.

[66] K. Tanaka, H. Sugiyama, T. Yamanari et al., "Renal expression of trefoil factor $3 \mathrm{mRNA}$ in association with tubulointerstitial fibrosis in IgA nephropathy," Nephrology (Carlton), vol. 23, no. 9, pp. 855-862, 2018.

[67] T. C. Spada, J. M. R. D. Silva, L. S. Francisco et al., "High intensity resistance training causes muscle damage and increases biomarkers of acute kidney injury in healthy individuals," PLoS One, vol. 13, no. 11, article e0205791, 2018.

[68] B. R. Griffin, S. Faubel, and C. L. Edelstein, "Biomarkers of drug-induced kidney toxicity," Therapeutic Drug Monitoring, vol. 41, no. 2, pp. 213-226, 2019.

[69] A. Zinellu, S. Sotgia, E. Sotgiu et al., "Cholesterol lowering treatment restores blood global DNA methylation in chronic kidney disease (CKD) patients," Nutrition, Metabolism, and Cardiovascular Diseases, vol. 27, no. 9, pp. 822-829, 2017. 


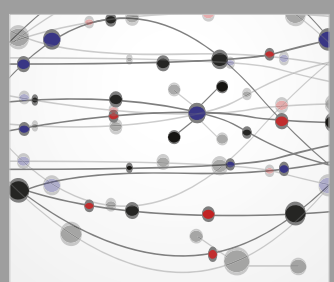

The Scientific World Journal
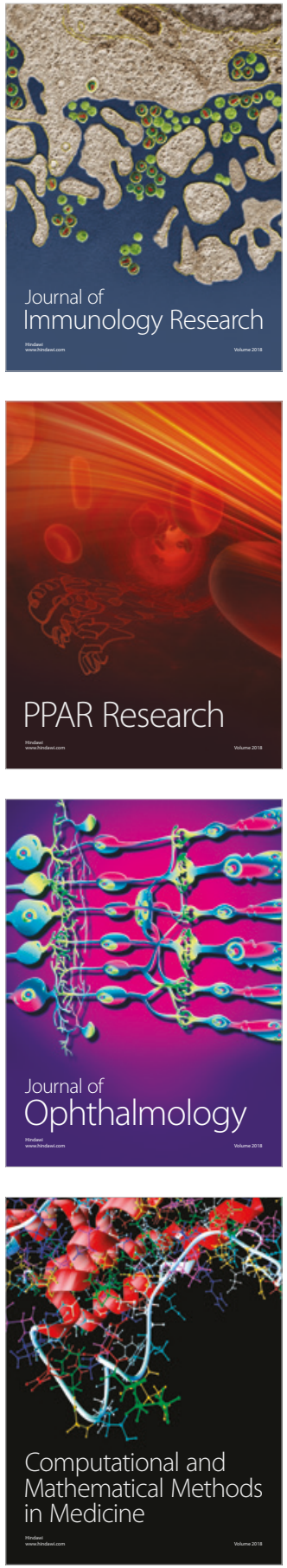

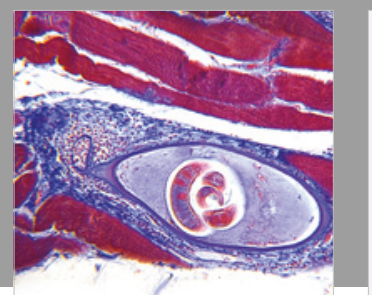

Gastroenterology Research and Practice

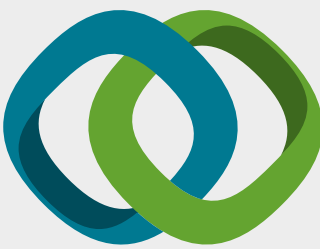

\section{Hindawi}

Submit your manuscripts at

www.hindawi.com
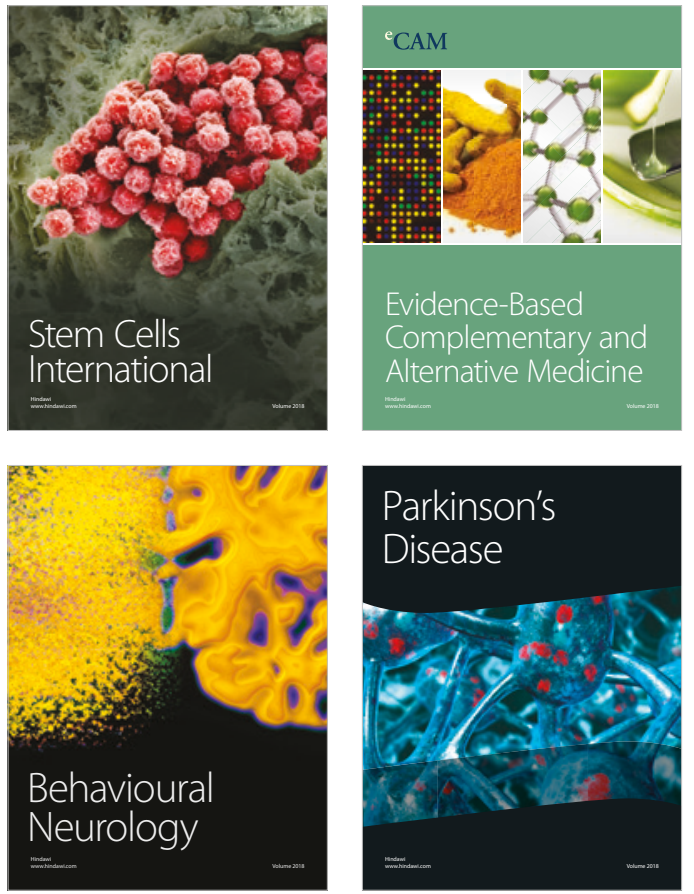

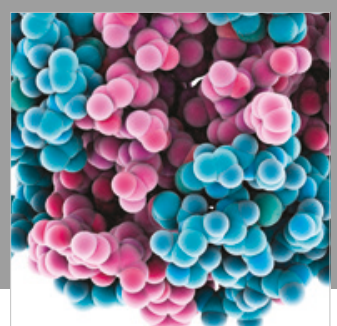

ournal of

Diabetes Research

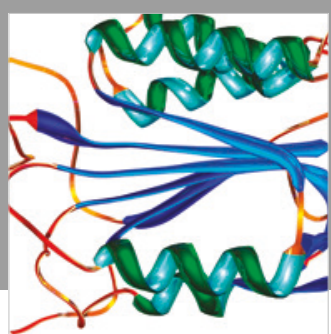

Disease Markers
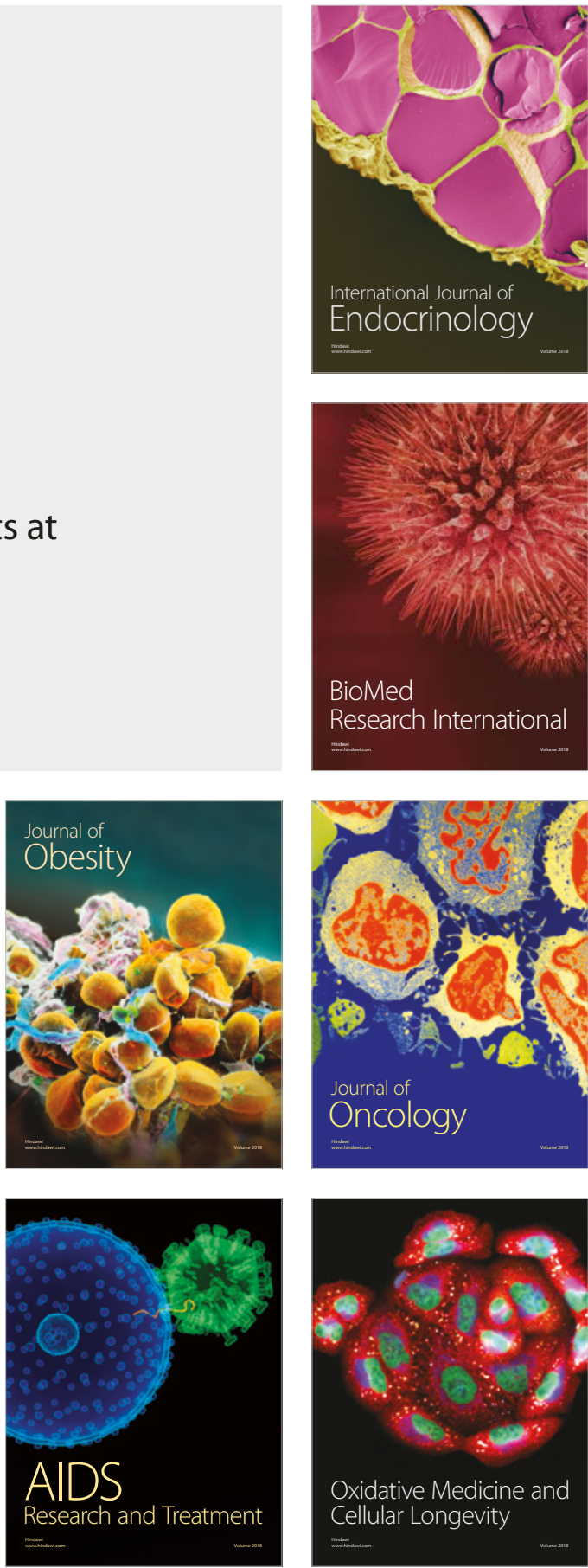